\title{
COMPARING A SINGLE-SENSOR CAMERA WITH A MULTISENSOR CAMERA FOR MONITORING COFFEE CROP USING UNMANNED AERIAL VEHICLES
}

\author{
Amanda P. A. Gomes ${ }^{1 *}$, Daniel M. de Queiroz ${ }^{1}$, Domingos S. M. Valente ${ }^{1}$, \\ Francisco de A. de C. Pinto ${ }^{1}$, Jorge T. F. Rosas ${ }^{2}$
}

\author{
${ }^{1 *}$ Corresponding author. Universidade Federal de Viçosa/ Departamento de Engenharia Agrícola/ Viçosa - MG, Brasil. \\ E-mail: amandapag@ hotmail.com | ORCID ID: https://orcid.org/0000-0002-8912-2690
}

\section{KEYWORDS}

precision agriculture, $\mathrm{UAV}$, radiometric calibration, modified RGB camera.

\begin{abstract}
There exist two options for digital cameras that can capture the near-infrared (NIR) band. Conventional red-green-blue (RGB, visible bands) cameras with a single sensor provide NIR band visibility based on the removal of the internal NIR-blocking filter. Alternatively, multisensor cameras exist that have a specific sensor for each band. The modified RGB cameras are of a lower price. In this context, the objective of this study was to compare the performance of a modified RGB camera with that of a multisensor camera for obtaining the normalized difference vegetation index (NDVI) in an area with coffee cultivations. A multispectral camera with five sensors and another camera with only one sensor were used. The NDVI of the coffee field was also measured using the GreenSeeker handheld NDVI sensor manufactured by Trimble. The images were calibrated radiometrically based on the targets in shades of gray made of napa, and the NDVI was calculated after image calibration. The calibration curves showed a high coefficient of determination. The NDVI value obtained with the calibrated images from the cameras showed a significant correlation with the values obtained by the GreenSeeker NDVI sensor, making it possible to obtain the variability pattern of the vegetation index. However, the NDVI obtained using the multisensor camera was closer to the NDVI obtained by the GreenSeeker NDVI sensor.
\end{abstract}

\section{INTRODUCTION}

The increase in coffee consumption around the world has made the importance of this crop even greater. Brazil is the largest coffee producing and exporting country (International Coffee Organization, 2019). However, because of international competition, it is necessary to develop strategies that can positively influence coffee production and quality. One of the alternatives to improve the efficiency of coffee production systems is the adoption of precision agriculture by coffee growers. The correct prescription and efficient use of agricultural inputs can be an alternative to meet the growing food demand and requirements for environmental sustainability. This can be achieved by applying inputs in variable dosages according to the spatial variability of the attributes associated with soil and plants (Mulla, 2013; Murugan et al., 2017).
To monitor the production fields so that they can be treated in a spatial variable fashion, remote sensing (RS) can be used. However, few studies have applied RS to coffee cultivation because of the difficulties imposed by this crop. The architecture and biomass of the trees cause some vegetation indices to lose sensitivity in the perception of chlorophyll (Manzano et al., 2019). The results obtained are better when working with images with higher spatial resolutions (Bernardes et al., 2012). The application of RS in areas with mountainous relief is more difficult because of the interaction of electromagnetic radiation with the relief. To monitor a crop and detect the spatial variability of production factors in a coffee field, multispectral images can be used. Multispectral images can be obtained using sensors coupled to aerial or orbital platforms. Satellite images are widely used for crop monitoring; however, their

\footnotetext{
${ }^{1}$ Universidade Federal de Viçosa/ Departamento de Engenharia Agrícola/ Viçosa - MG, Brasil.

${ }^{2}$ Universidade de São Paulo/ Escola Superior de Agricultura "Luiz de Queiroz"/ Departamento de Solos e Nutrição de Plantas/ Piracicaba - SP, Brasil.
} 
use depends on the temporal resolution, spatial resolution, and spectral resolution of the sensor (Corti et al., 2019).

The use of unmanned aerial vehicles (UAVs) for agricultural purposes is growing. One of their advantages is the flexibility in scheduling data collection, because it depends only on the availability of the operator and environmental conditions. These devices are equipped with multispectral digital cameras that are characterized by the ability to obtain images with high temporal and spatial resolutions. The cameras are coupled to the UAVs and used to obtain periodic crop spectral information. Information on the development of vegetation from images is generally based on indices that compare the reflectance of vegetation in more than one spectral band (Nijland et al., 2014). The most common indices use the difference in the reflectance of the targets between the near-infrared (NIR) and red (R) bands or other bands of the spectrum (Hunt et al., 2011; Nijland et al., 2014), as is the case of the normalized difference vegetation index (NDVI), which is applied when investigating the vigor status of the crop.

Commercial cameras generally work in the visible band, and sensors that can acquire NIR image data tend to be more expensive, because of which these are not adopted extensively (Hunt et al., 2011; Logie \& Coburn, 2018). Some of these cameras are modified by the application of filters to acquire data in specific bands, such as the NIR band. The modification of red-green-blue (RGB) cameras involves removing the NIR blocking filter (Hunt et al. 2010; Lebourgeois et al., 2008). However, sensors to assess the condition of vegetation must undergo a calibration whereby the radiometric behavior of each pixel is analyzed in the different regions of the spectrum where the information was recorded (Corti et al., 2019; Pozo et al., 2014; Putra \& Soni, 2017). This process depends on the characteristics of the sensor and climatic conditions, including the variation of ambient light (Wang \& Myint, 2015). Thus, radiometric calibration is essential when working with multispectral images.

However, the price of multispectral cameras is one of the limiting factors for their use in agriculture. Their prices rise with the increase in the number of spectral bands and with an increase in spatial resolution. The number of bands that the camera collects determines which and how many vegetation indices can be obtained. Therefore, the objective of this study was to compare the performance of a single-sensor camera with that of a multisensor camera in obtaining the NDVI in areas cultivated with coffee in a mountainous region.

\section{MATERIAL AND METHODS}

The experiment was carried out at Fazenda Jatobá, located in the municipality of Paula Cândido, Minas Gerais (Figure 1). At Fazenda Jatobá, coffee of the species Coffea arabica is grown. The study area has a mountainous relief with a total area of 65 ha.

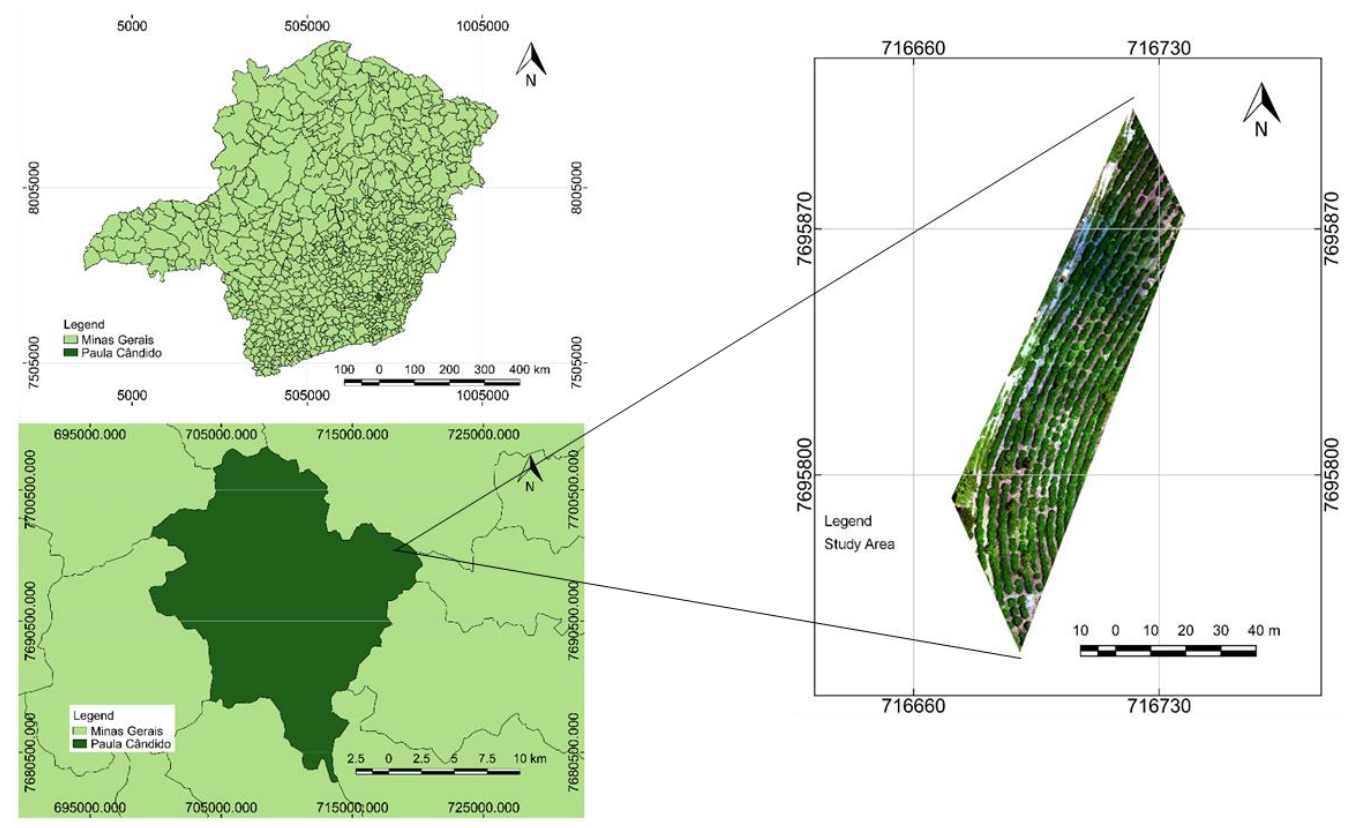

FIGURE 1. Map showing the study area, Jatobá Farm, Paula Cândido-MG.

Two multispectral cameras and two UAVs were used for image acquisition. One of the UAVs used was the Matrice 100 model (SZ DJI Technology Co., Shenzhen, Guangdong, China) with rotating propellers and an electric drive (Figure 2A). The second UAV was the Phantom 4 Pro model (SZ DJI Technology Co., Shenzhen, Guangdong,
China), shown in Figure 2B. This model has a system with vision sensors to detect front, rear, right, and left obstacles. For aerial control of the UAV, an earth station at a strategic point in the experimental area was used. Flight control was carried out by means of radio control with the routes and camera firing previously defined in the flight plan. 


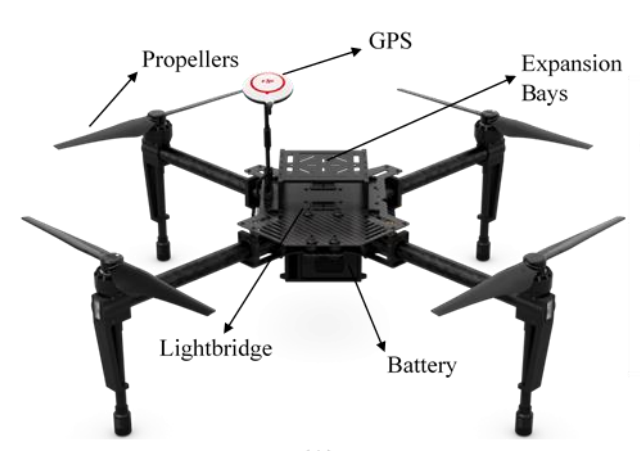

(A)

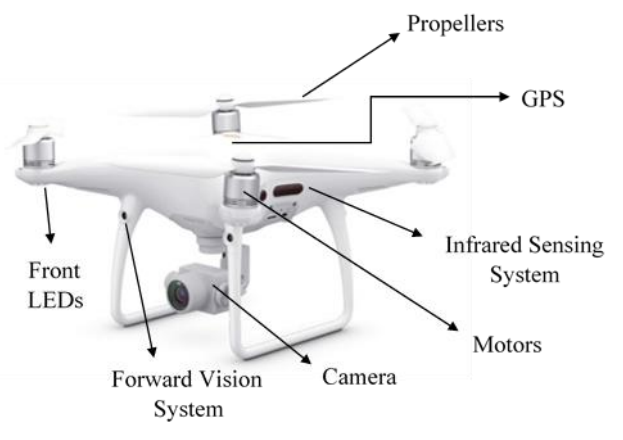

(B)

FIGURE 2. Unmanned aerial vehicle used for image acquisition: (A) Matrice 100 and (B) Phantom 4 Pro (source: adapted from SZ DJI Technology Co.).

The cameras used were a MAPIR Survey3W (MAPIR, Peau Productions, Inc., CA, USA), shown in Figure 3B, and MicaSense RedEdge-MX (MicaSense, Inc., Seattle, WA, USA), shown in Figure 3A. The MAPIR was coupled to the Phantom, while the MicaSense was installed in the Matrice 100. The MAPIR Survey3W camera is a modified camera that collects images in the $\mathrm{R}$ and green bands (centered at 660 and $550 \mathrm{~nm}$, respectively) and the NIR band (centered at $850 \mathrm{~nm}$ ). The sensor of the camera has 12 megapixels, with a resolution of $4032 \times 3024$, and it is capable of producing images in RAW (12-bit) and JPG (8-bit) formats. The MAPIR camera settings were fixed using custom 10/10 for white balance, 100 for ISO, 1/500

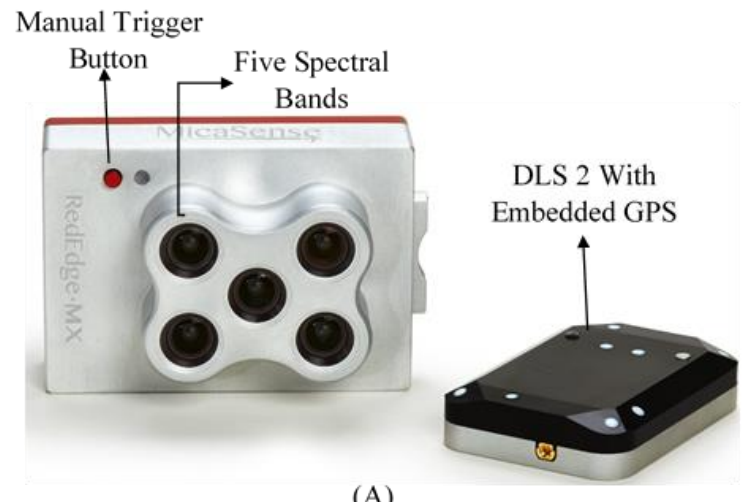

(A) shutter aperture, and +0.0 exposure. The RedEdge-MX MicaSense camera has a metallic structure and collects images in the R (centered at $668 \mathrm{~nm}$, bandwidth of $14 \mathrm{~nm}$ ), green (centered at $560 \mathrm{~nm}$, bandwidth of $27 \mathrm{~nm}$ ), blue (centered at $475 \mathrm{~nm}$, width $32 \mathrm{~nm}$ bandwidth), red edge (centered at $717 \mathrm{~nm}$, bandwidth $12 \mathrm{~nm}$ ), and NIR (centered at $842 \mathrm{~nm}$, bandwidth $57 \mathrm{~nm}$ ) bands. It can produce images in RAW format (12 bit) and TIFF (16 bit). It has a DSL-2 module that measures irradiance and the angle of the sun's rays, and it has an integrated Global Navigation Satellite System sensor. The MicaSense RedEdge-MX settings are regulated by the camera itself according to the ambient lighting determined by the DSL- 2 module.

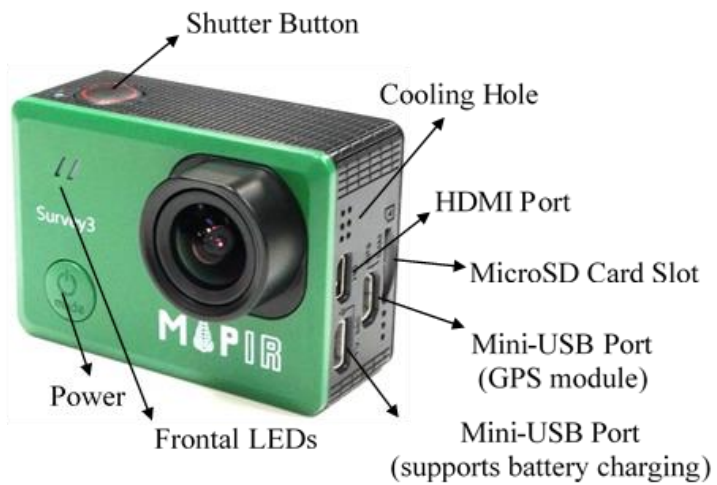

(B)

FIGURE 3. Cameras used for image acquisition: (A) MicaSense RedEdge-MX and (B) MAPIR Survey3W (source: adapted from MicaSense and MAPIR).

Figure 4 depicts the workflow of the proposed methodology for collecting and processing the images. Four control points were installed in the area, in addition to 36 experimental points. The experimental points were the locations where data collection was performed. These points were randomly distributed in the area, and they defined the points on the coffee plants and between the crop lines for data acquisition. All points were georeferenced using the PRO-XT model (Trimble Navigation Ltd., Sunnyvale, CA, USA), and postprocessed differential correction was applied. At each experimental point, the NDVI was determined with the aid of the GreenSeeker handheld crop sensor (Trimble Navigation Ltd., Sunnyvale, CA, USA), as shown in Figure 5. The NDVI was determined by calculating the average of three readings taken with the GreenSeeker sensor. The readings were performed by placing the sensor above the coffee plants. The distance between the sensor and the plant was maintained at $30 \mathrm{~cm}$. When the NDVI was measured between the crop lines, the sensor was kept $30 \mathrm{~cm}$ above the ground. 


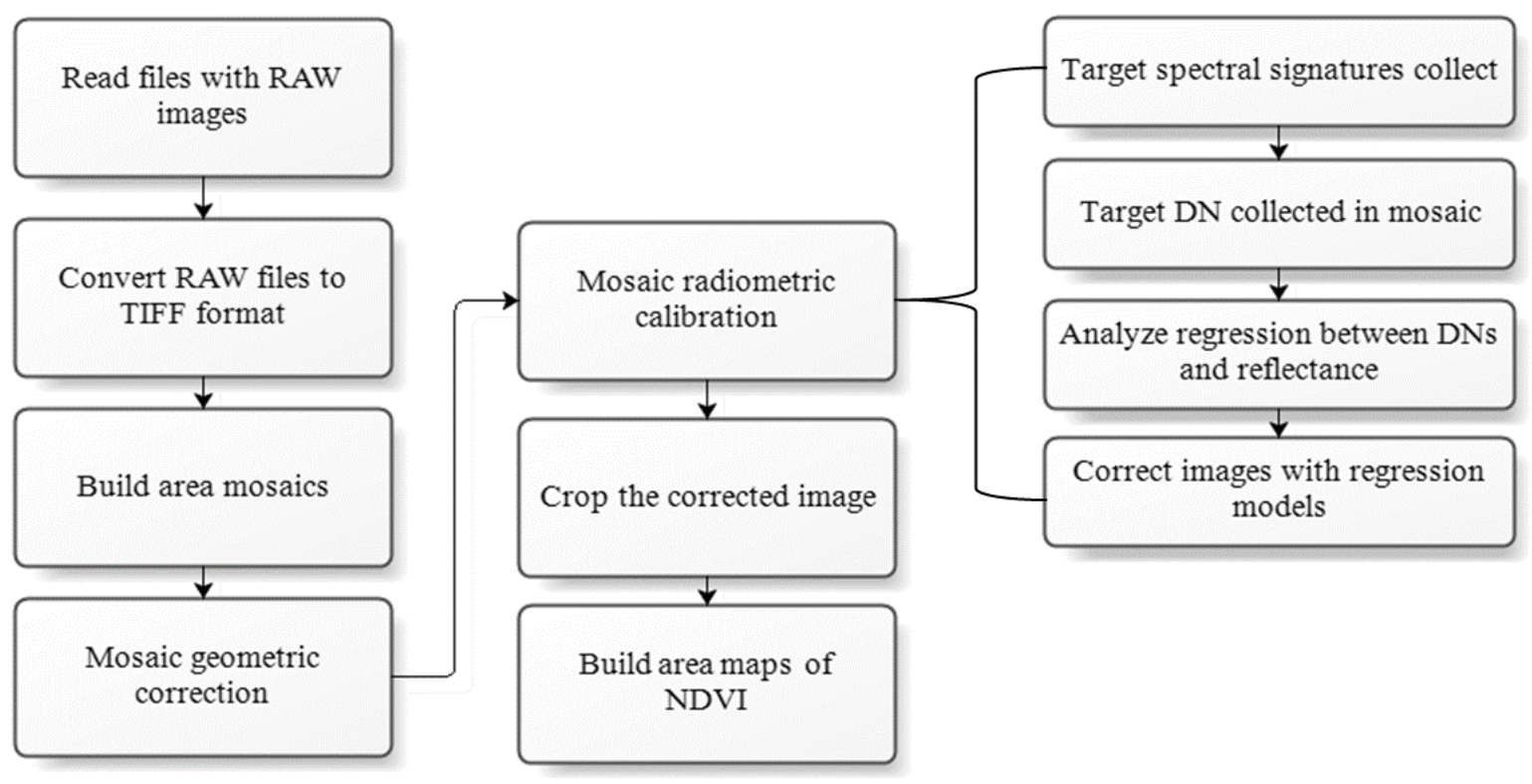

FIGURE 4. Flowchart of the data processing.

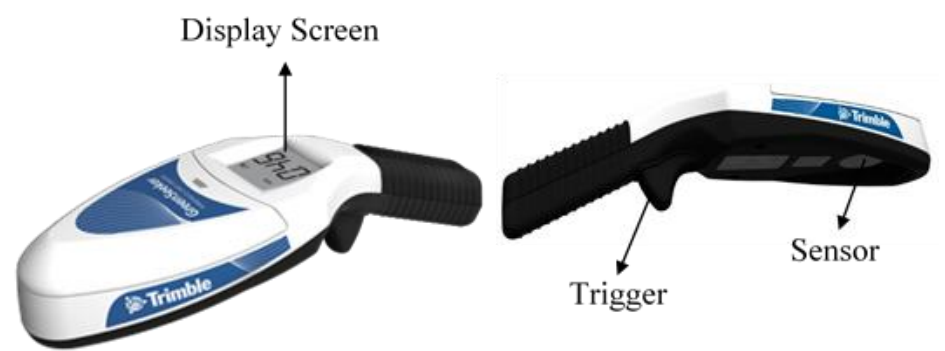

FIGURE 5. GreenSeeker handheld NDVI crop sensor (source: adapted from Trimble).

The images were collected on three different days, always at 11:00 a.m. The days of the image collections took an interval of 20 days, with the first collection taking place in November, the second taking place in December, and the third taking place in January. The flights were performed at an altitude of $50 \mathrm{~m}$, guaranteeing a spatial resolution of approximately $2.5 \mathrm{~cm}$ for the images from the MAPIR Survey $3 \mathrm{~W}$ camera and $3.5 \mathrm{~cm}$ for the images from the MicaSense RedEdge-MX camera, as well as an overlapping of longitudinal and lateral images of $75 \%$ for both cameras.

The images obtained with the Survey3W MAPIR camera were submitted through the process of converting the format from RAW to TIFF with the aid of the MAPIR Camera Control software. For data processing purposes, at the time the images were converted, this software caused the radiometric resolution of the pixels to be changed from 12 to 16 bits. This change did not result in loss of information acquired at the time of image acquisition because there was a filling with four zero bits in each digital number (ND) of the image. MicaSense RedEdge-MX also performs this filling when saving the TIFF images in 16 bits.
Using the images from each camera, the mosaics were generated using the Agisoft Metashape Professional software version 1.5.0 (developed by Agisoft LLC, Russia). The mosaic generation process consisted of aligning the images, building a densified cloud, building a model and texture, and building a digital elevation model to complete the database and generate the mosaic. Then, these mosaics were georeferenced through the collected control points and the geographic information system (GIS) QGIS (Quantum GIS, developed by the QGIS Development Team), version 2.18, using the georeferencing tool.

For radiometric calibration, targets made of napa (a fabric composed of laminated polyvinyl chloride and polyester) with dimensions of $1.00 \times 1.50 \mathrm{~m}$ in white, black, light gray, and dark gray were used. These targets were arranged in the area during the flights (Figure 6). In the laboratory, the spectral signature (Figure 7) of each target was obtained with the aid of the ASD FieldSpec HandHeld 2 spectroradiometer (Analytical Spectral Devices, Inc., Boulder, CO, USA) (Figure 8). A measurement was performed with five repetitions at random points for each target, and the average was calculated. 


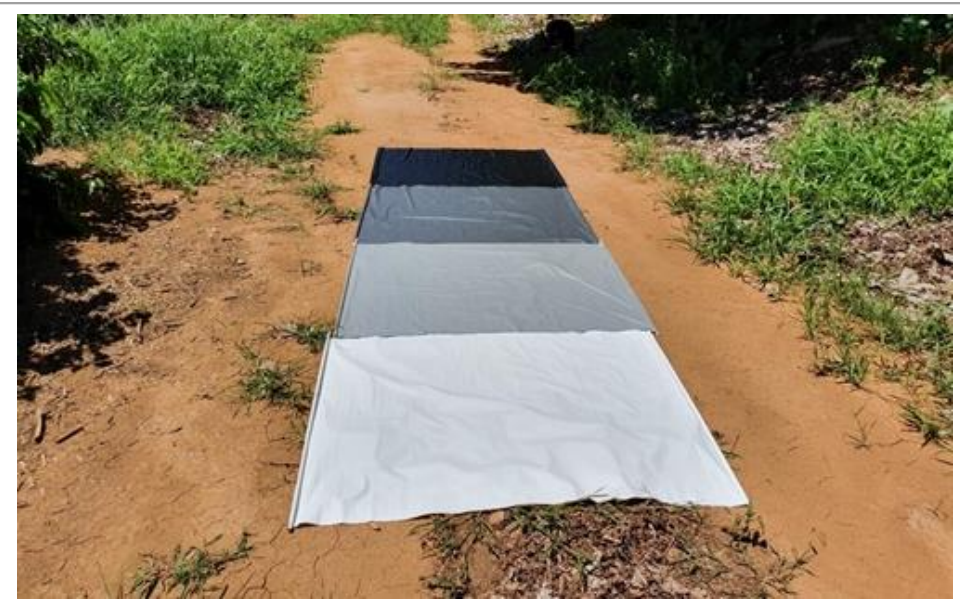

FIGURE 6. Calibration targets placed on the ground during the test days.

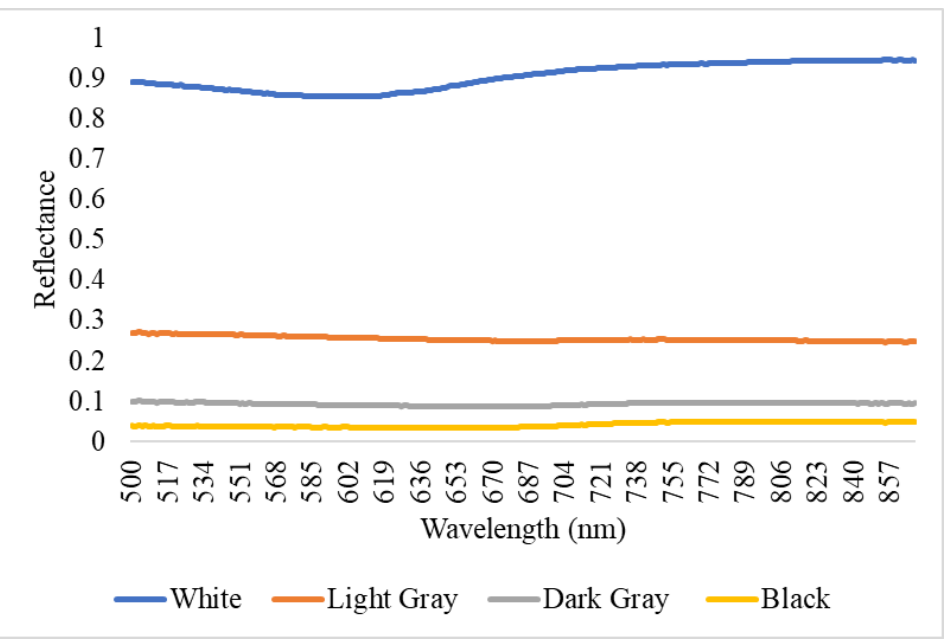

FIGURE 7. Spectral signature of napa fabric targets in white, light gray, dark gray and black for radiometric calibration.

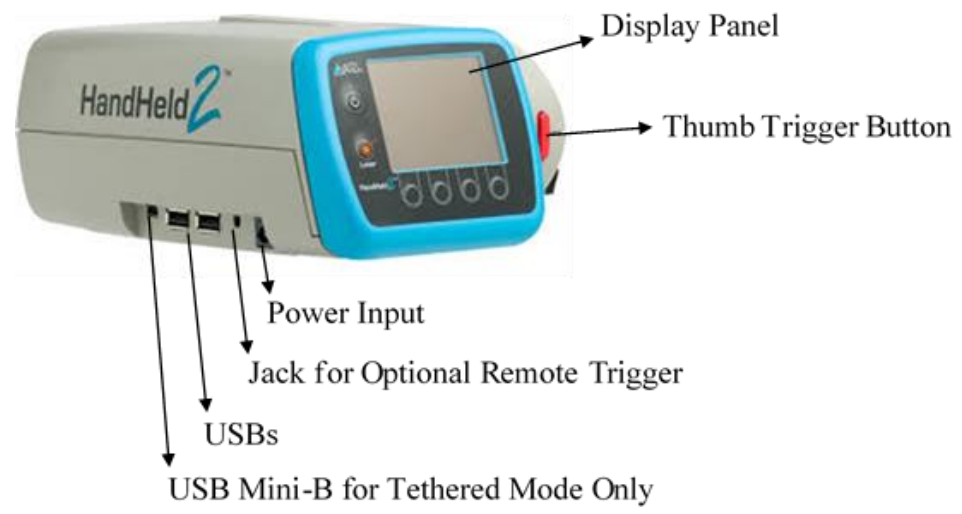

FIGURE 8. ASD FieldSpec HandHeld Spectroradiometer 2 (source: adapted from Malvern Panalytical).

The radiometric calibration of the images consisted of generating regressions using the image $\mathrm{DN}$ of the calibration targets with their respective reflectance values. The central NDs of each target were collected, and an average was calculated for each target and the R and NIR bands, using the statistical tool by zones of the QGIS software. Using an electronic spreadsheet, these averages, along with the respective reflectance of the target in each waveband (Figure 7), were used to generate regressions. Regressions were generated for the $\mathrm{R}$ and NIR bands for each collection day. The area of the coffee field was cut using the QGIS cut tool. With the regression results, it was possible to carry out the radiometric calibration of each band of the mosaics and estimate the NDVI using the QGIS raster calculator tool. Thus, the NDVI obtained based on the images of the cameras was compared with the NDVI obtained using GreenSeeker as a reference (Bourgeon et al., 2016; Cao et al., 2019).

\section{RESULTS AND DISCUSSION}

The calibration equations and the determination coefficient for the R and NIR bands can be seen in Figure 9. The average reflectance for the R and NIR bands and the root mean squared error (RMSE) for the calibration targets are shown in Table 1 . The relationship between the 
reflectance of the calibration target and the NDs of the image varies depending on the sensor used and the type of calibration target.

Linear regressions were applied for both bands with the MAPIR Survey3W images, whereas, for the MicaSense RedEdge-MX camera, the R band showed an exponential behavior. The same phenomenon occurred in the study of Deng et al. (2018). They found exponential and linear curves using gray-scale targets, but the sensor used in the study was the Mini-MCA made by Tetracam (Tetracam, Inc., Chatsworth, CA, USA). However, Guo et al. (2019) recommended the use of a linear relationship between the reflectance and ND of the targets when using the MiniMCA 6 camera, also made by Tetracam, Inc. They also recommended the use of three different targets for radiometric calibration: dark, moderate, and white calibration targets.

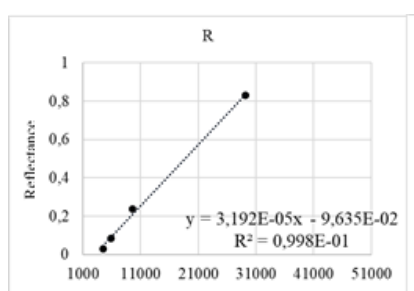

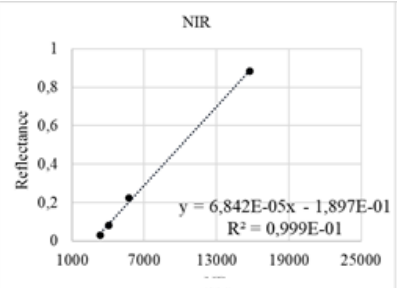
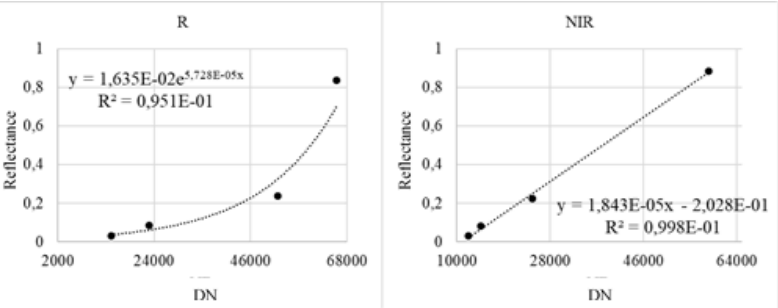

DN

Day 1
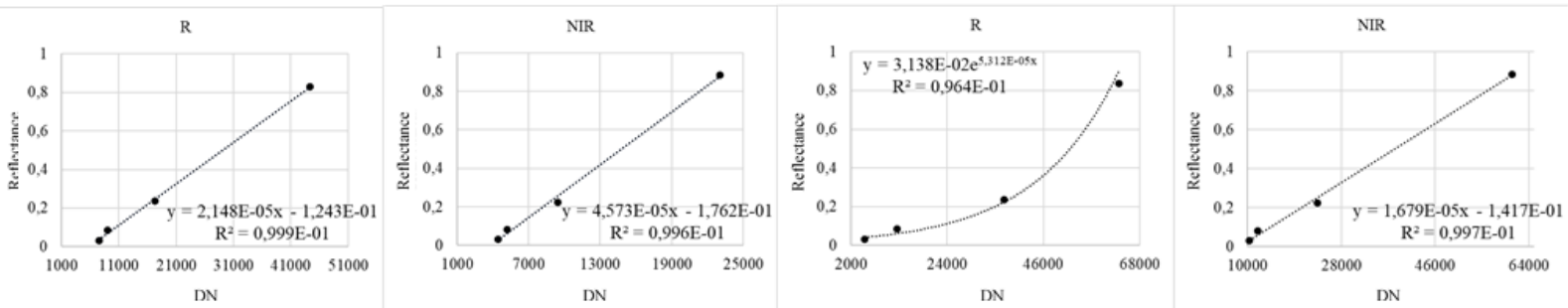

DN

Day2
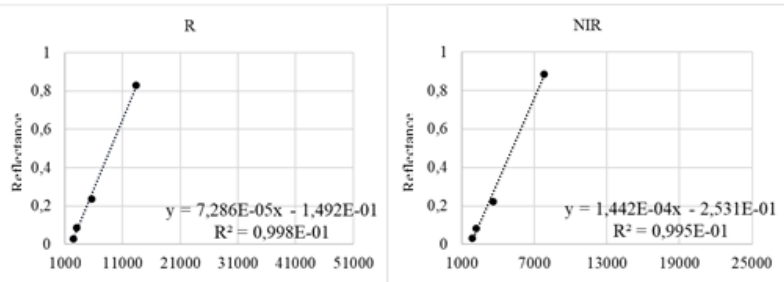

DN

(A)
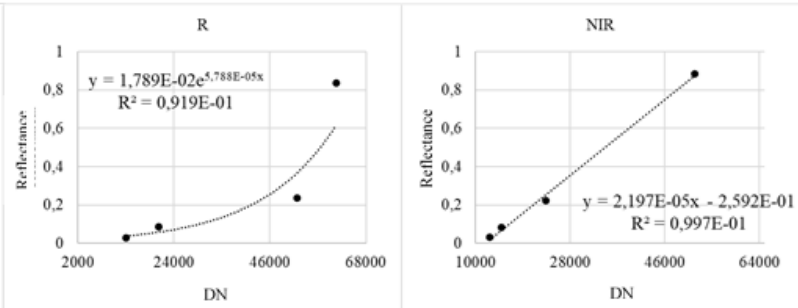

(B)

FIGURE 9. Calibration equations and determination coefficient for the red (R) and near-infrared (NIR) bands for (A) MAPIR Survey $3 \mathrm{~W}$ and (B) MicaSense RedEdge-MX.

TABLE 1. Mean and mean square reflectance error for the red and near-infrared bands.

\begin{tabular}{cc}
\hline & MAPIR Survey3W \\
\hline RMSE & Mean Reflectance \\
\hline $1.564 \mathrm{E}-02$ & 0.316 \\
$1.106 \mathrm{E}-02$ & 0.334 \\
$1.003 \mathrm{E}-02$ & 0.316 \\
$2.064 \mathrm{E}-02$ & 0.334 \\
$1.264 \mathrm{E}-02$ & 0.316 \\
$2.416 \mathrm{E}-02$ & 0.334 \\
\hline \multicolumn{2}{c}{ MicaSense RedEdge-MX } \\
\hline RMSE & Mean Reflectance \\
\hline 8.421E-02 & 0.317 \\
$1.624 \mathrm{E}-02$ & 0.334 \\
$3.418 \mathrm{E}-02$ & 0.317 \\
$1.785 \mathrm{E}-02$ & 0.334 \\
$1.293 \mathrm{E}-01$ & 0.317 \\
$1.824 \mathrm{E}-02$ & 0.334 \\
\hline
\end{tabular}

The relationship between ND and reflectance depends on the sensor used as well as the types and colors of the targets. In the literature, it is possible to find relations that are not only linear and exponential, but also polynomial, such as that reported by Crusiol et al. (2017). They obtained a second-degree polynomial relationship when working with Fujifilm S200-EXR cameras and fabric targets in white, black, gray, yellow, red, and green. Another aspect is that, when the camera does not have a linear response, modified empirical methods can be applied using more than two targets. For instance, Wang \& Myint (2015) used nine tones of gray to model an exponential relationship between the ND of a modified single-lens RGB camera and the target reflectance for imaging in the NIR band.

For both cameras, the coefficient of determination of the calibration regression between the ND and the reflectance obtained in the laboratory for the $\mathrm{R}$ and NIR bands was greater than $91 \%$. This result demonstrates that the calibration equations showed a good fit. The coefficients of determination obtained when using MAPIR Survey3W, in most cases, were higher than those obtained when using MicaSense RedEdge-MX. This may be caused by the way both cameras work. The MAPIR does not have a light sensor as the MicaSense camera does. This light sensor may 
interfere in different ways once the four targets have different reflectances. On the third day of image acquisition, unlike the other two, the day was cloudy, and this influenced the adjustment of the calibration curve. On that day, the RMSE obtained with MAPIR Survey3W was higher for the NIR band than on the other two days, and one of the lowest values was for the $\mathrm{R}$ band. This is because, in cameras that have only one sensor, it is not possible to adjust the parameters separately for each band. Therefore, the camera settings used favored the $\mathrm{R}$ band on the third day of the survey, but the settings were not good for the NIR band.

Figure 10 shows that higher values of NDVI were generated when using the GreenSeeker sensor. MAPIR Survey $3 \mathrm{~W}$ calibrated images tend to result in lower values and less spatial variability of the NDVI, whereas calibrated images from the MicaSense RedEdge-MX camera tend to show greater spatial variability in NDVI values. The MAPIR Survey3W camera consists of only one sensor to sense the three bands, so the adjustment of the camera settings is not done separately for each band - that is, the same configuration is used to capture the three bands. For this reason, the settings can result in a good adjustment for one band and be not as good for the other two bands. Then, the reflectance may not be estimated well for these two bands. The lower spatial variability of the NDVI obtained by the MAPIR Survey3W camera may be caused by this lack of establishing individual settings for each band.

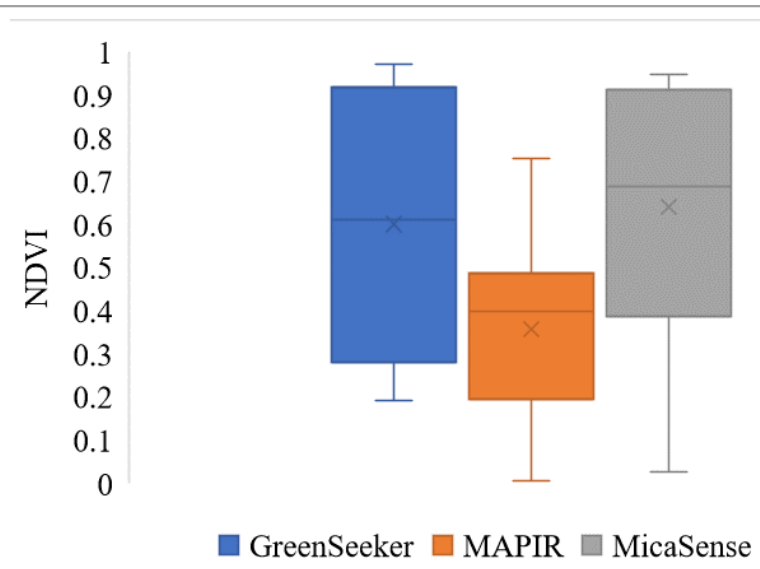

FIGURE 10. Comparison of the NDVI distribution obtained by the sensors for the three days of data collection.

Figure 11 shows the NDVI maps for each day of collection and for each camera used. The images reveal the coffee planting lines as they present colors from orange to green, symbolizing higher NDVI values. As the maps show, the NDVI values obtained with the radiometric calibration of the MicaSense RedEdge-MX images were higher than those calculated from the calibrated images of the MAPIR Survey $3 \mathrm{~W}$ camera. On the third day, the areas between lines indicated lower NDVI values because the farm owner had performed weed control. 


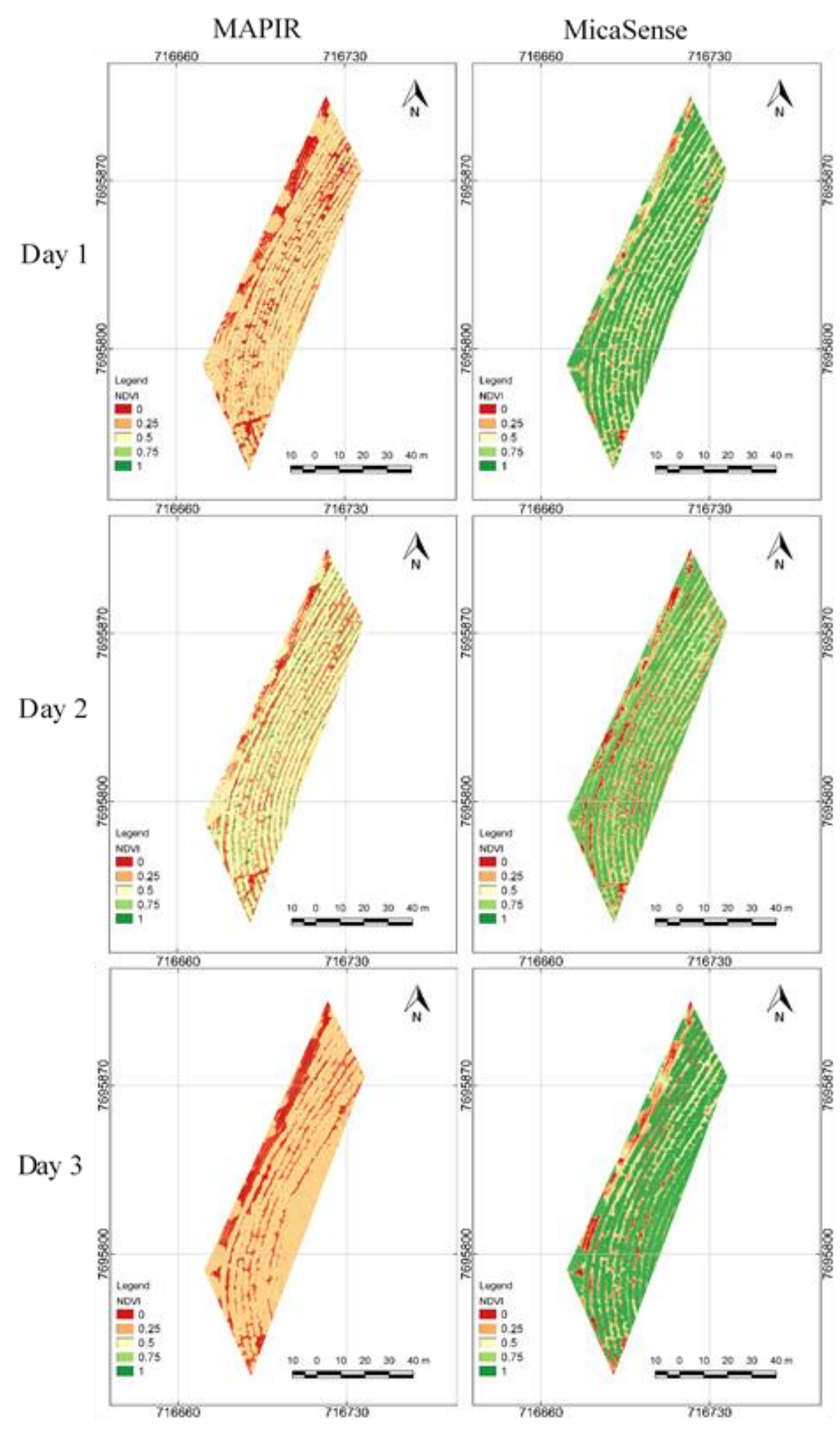

FIGURE 11. NDVI map obtained with the radiometric calibration of MAPIR Survey3W and MicaSense RedEdge-MX cameras.

Figure 12 shows the NDVI behavior at the experimental points for each day according to the GreenSeeker sensor, MAPIR Survey3W, and MicaSense RedEdge-MX. Points numbered using odd numbers represent points on the planting lines, while points numbered with even numbers represent points between lines. Points 29 to 36 were located near the edge of the plantation, where there was shading caused by the surrounding trees, so their NDVI values changed because of the shading. Moreover, the NDVI values obtained with the calibration of the MAPIR Survey3W images were lower than those obtained with the GreenSeeker sensor and with the calibrated images from the MicaSense RedEdge-MX camera. The MicaSense RedEdge-MX images, after image calibration, resulted in NDVI values close to those obtained with the GreenSeeker sensor. 


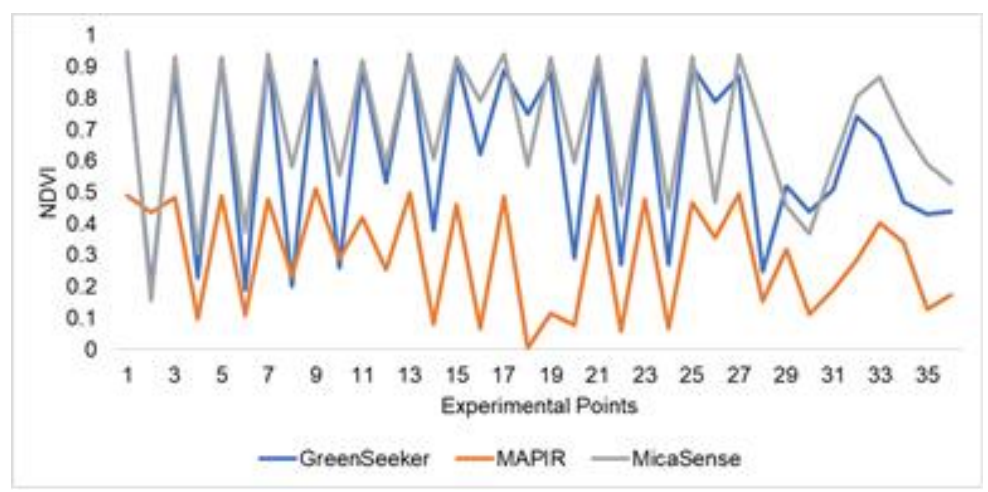

(a)

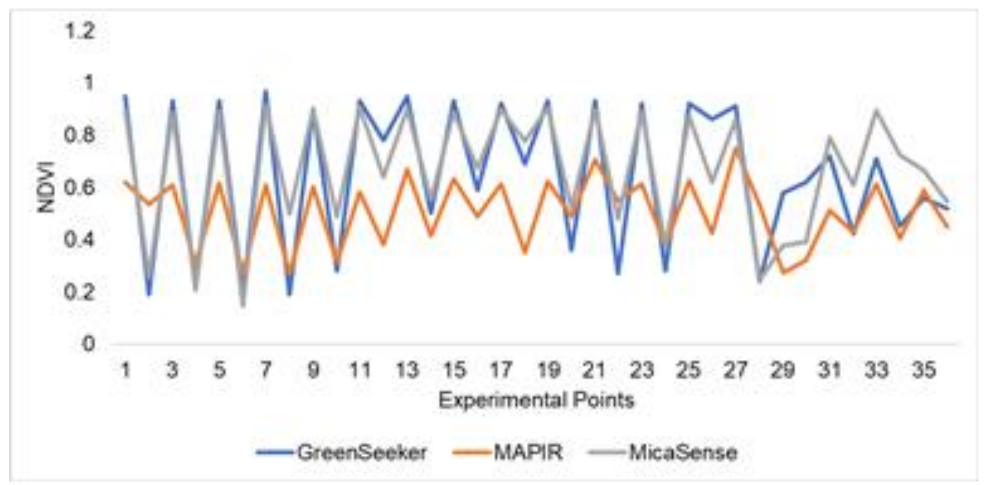

(b)

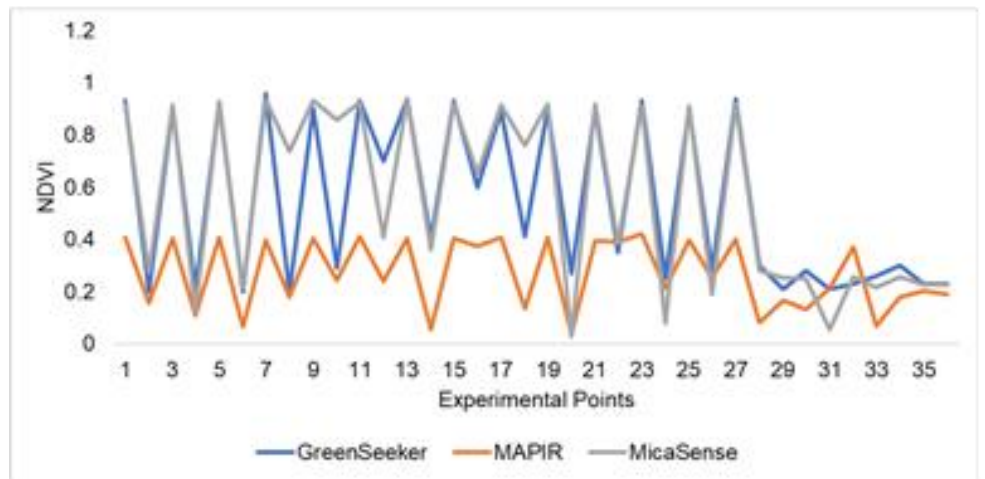

(c)

FIGURE 12. NDVI behavior among the three sensors used, GreenSeeker, MAPIR Survey3W, and MicaSense RedEdge-MX: (a) day 1 , (b) day 2 , and (c) day 3

Some reasons can be found to explain why the behavior of the NDVI values obtained from images acquired by the MAPIR Survey3W camera was inferior to those obtained by the MicaSense RedEdge-MX camera. One is that the incident solar radiation on the calibration targets was different from the incident solar radiation on the 36 points where the measurement with the GreenSeeker sensor was performed. The calibration targets were located on a road that was a flat area located on the boarder of the coffee field and not inside the coffee field.

Another possible cause for the lower values of NDVI obtained with the MAPIR Survey3W camera may be associated with the characteristics of the camera. One problem is that the manufacturer does not disclose the response curve of each band for the sensor in the MAPIR cameras. However, it is known that this camera is equipped with a single sensor to capture images in the three bands. Thus, the signal of each band may be affected by the shutter opening, which cannot be adjusted for each band, as well as the other parameters of the camera. As a result, the electromagnetic radiation spectrum processed by the sensor may interfere with the ND value obtained for each band. According to Nijland et al. (2014), in modified cameras, the transmission profiles of colored channels, after removing the filter, remain sensitive to infrared radiation. Therefore, in the R band, the MAPIR Survey $3 \mathrm{~W}$ camera captures not only the energy in the wavelength of the R band, but also a part of the NIR (Nijland et al., 2014), which generates an increase in the reflectance of the $\mathrm{R}$ band and a reduction in the obtained NDVI values.

The targets used in the radiometric calibration process had practically the same reflectance in the NIR, green, and $R$ bands (Figure 7). Because the vegetation has a reflectance in the NIR region that is greater than that in the R band (Figure 13), the radiometric calibration by the empirical line method may interfere with the reflectance values in the $\mathrm{R}$ and infrared bands, causing an error in determining the NDVI of the vegetation by the MAPIR Survey3W. 


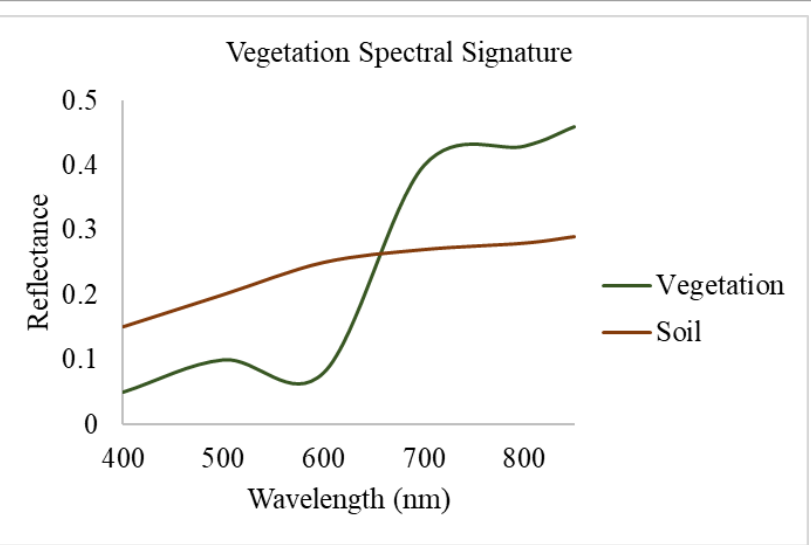

FIGURE 13. Spectral behavior of vegetation and soil (source: adapted from INPE).

The behavior of the NDVI obtained from the images collected by the MAPIR Survey3W camera was similar to that obtained by the GreenSeeker sensor and the MicaSense RedEdge-MX camera (Figure 12). The MAPIR camera response was better in determining the NDVI of the points between the lines, which had a lower density of vegetation, than of the coffee trees, reinforcing the hypothesis that the characteristics of the MAPIR Survey3W camera are the cause of the lower NDVI values. The explanation is that the reflectance values of the soil in the $\mathrm{R}$ and infrared bands are closer than those of a coffee plant (Figure 13). This behavior is similar to that found in the targets used in radiometric calibration (Figure 7).

Table 2 shows the correlations between the NDVIs obtained with the GreenSeeker NDVI sensor and calibrated images from the MAPIR Survey $3 \mathrm{~W}$ and MicaSense RedEdge-MX cameras. Even though there is a difference between the NDVI values obtained by the two cameras and by the GreenSeeker sensor, Table 2 shows that the NDVI values obtained by the three methods have a significant correlation. In the case of the MAPIR Survey $3 \mathrm{~W}$ camera, the NDVI calculated after calibrating the images showed a significant correlation, but with a tendency of lower values compared with the other sensors, except for the third day. On that day, the weather was cloudy, and, in this condition, a higher correlation coefficient value was obtained between the NDVI measured using the GreenSeeker sensor and the NDVI obtained based on the MAPIR Survey3W.

TABLE 2. Correlation between the NDVI obtained with the GreenSeeker sensor, MAPIR Survey3W camera, and MicaSense RedEdge-MX camera.

\begin{tabular}{cccc}
\hline \multicolumn{4}{c}{ Day 1 } \\
\hline & GreenSeeker & MAPIR & MicaSense \\
\hline GreenSeeker & 1 & $0.636^{*}$ & $0.803^{*}$ \\
MAPIR & - & 1 & $0.711^{*}$ \\
MicaSense & - & - & 1 \\
\hline \multicolumn{4}{c}{ Day 2 } \\
\hline GreenSeeker & MAPIR & MicaSense \\
\hline GreenSeeker & 1 & $0.726^{*}$ & $0.896^{*}$ \\
MAPIR & - & 1 & $0.786^{*}$ \\
MicaSense & - & - & 1 \\
\hline \multicolumn{5}{c}{ Day 3 } \\
GreenSeeker & GreenSeeker & MAPIR & MicaSense \\
MAPIR & 1 & $0.930^{*}$ & $0.895^{*}$ \\
MicaSense & - & 1 & $0.913^{*}$ \\
\hline
\end{tabular}

*significant at 5\% level
To obtain the crop NDVI spatial variability pattern, the MAPIR Survey3W camera can be used. However, this camera cannot monitor the NDVI temporal variability; in this case, the MicaSense RedEdge-MX is the recommended camera. Moreover, the MAPIR Survey $3 \mathrm{~W}$ captures a smaller number of bands, making it possible to obtain a smaller number of vegetation indices. The MicaSense RedEdge-MX, which has a greater number of bands, can obtain a greater number of vegetation indices; however, it is a camera that has a much higher price than the MAPIR Survey3W.

\section{CONCLUSIONS}

Digital cameras are promising tools not only for crop monitoring, but also for several other purposes. Modified cameras, such as the MAPIR Survey3W, are not suitable for studies on crop temporal variability monitoring, because the calculated value of the NDVI should not be trusted. Multisensor cameras, such as the MicaSense RedEdge-MX, make it possible to obtain an NDVI that is closer to the ones determined by the GreenSeeker sensor. They have a better performance for working with crop spatial and temporal monitoring, in addition to presenting a greater number of bands, which makes it possible to work with a greater number of vegetation indices.

\section{ACKNOWLEDGMENTS}

The authors would like to acknowledge the support of the Coordination for the Improvement of Higher Education Personnel, Brazil (CAPES), Financing Code 001, the National Council for Scientific and Technological Development, Brazil (CNPq), and the Research Support Foundation of the State of Minas Gerais (FAPEMIG).

\section{REFERENCES}

Bernardes T, Moreira MA, Adami M, Giarolla A, Rudorff BFT (2012) Monitoring biennial bearing effect on coffee yield using MODIS remote sensing imagery, Remote Sensing 4(9):2492-2509. DOI: https://doi.org/10.3390/rs4092492

Bourgeon MA, Paoli JN, Jones G, Villette S, Gée C (2016) Field radiometric calibration of a multispectral on-the-go sensor dedicated to the characterization of vineyard foliage, Computers and Electronics in Agriculture 123:184-194. DOI: https://doi.org/10.1016/j.compag.2016.02.019 
Cao S, Danielson B, Clare S, Koenig S, Vargas CC, Azofeifa AS (2019) Radiometric calibration assessments for UAS-borne multispectral cameras: Laboratory and field protocols, ISPRS Journal of Photogrammetry and Remote Sensing 149:132-145. DOI:

https://doi.org/10.1016/j.isprsjprs.2019.01.016

Corti M, Cavalli D, Cabassi G, Vigoni A, Degano L, Gallina PM (2019) Application of a low-cost camera on a UAV to estimate maize nitrogen-related variables. Precision Agriculture 20(4): 675-696. DOI: https://doi.org/10.1007/s11119-018-9609-y

Crusiol LGT, Nanni MR, Silva GFC, Furlanetto RH, Gualberto AAS, Gasparotto AC, De Paula MN (2017) Semi professional digital camera calibration techniques for Vis/NIR spectral data acquisition from an unmanned aerial vehicle. International Journal of Remote Sensing 38(810):2717-2736. DOI:

https://doi.org/10.1080/01431161.2016.1264032

Deng L, Hao X, Mao Z, Yan Y, Sun J, Zhang A (2018) A Subband Radiometric Calibration Method for UAV-Based Multispectral Remote Sensing. IEEE Journal of Selected Topics in Applied Earth Observations and Remote Sensing 11(8): 2869-2880. DOI:

https://doi.org/10.1109/JSTARS.2018.2842466

Guo Y, Senthilnath J, Wu W, Zhang X, Zeng Z, Huang H (2019) Radiometric calibration for multispectral camera of different imaging conditions mounted on a UAV platform. Sustainability (Switzerland) 11(4): 978. DOI: https://doi.org/10.3390/su11040978

Hunt ER, Hively WD, Fujikawa SJ, Linden DS, Daughtry CST, McCarty GW (2010) Acquisition of NIR-green-blue digital photographs from unmanned aircraft for crop monitoring. Remote Sensing 2(1):290-305. DOI: https://doi.org/10.3390/rs2010290

Hunt ER, Hively WD, McCarty JW, Daughtry CST, Forrestal PJ, Kratochvil RJ, Carr JL, Allen NF, FoxRabinovitz JR, Miller CD (2011) NIR-green-blue highresolution digital images for assessment of winter cover crop biomass. GIScience and Remote Sensing 48(1): 8698. DOI: https://doi.org/10.2747/1548-1603.48.1.86

International Coffee Organization (2019) Coffee development 2019 Report: growing for prosperity. International Coffee Organization.

Lebourgeois V, Bégué A, Labbé S, Mallavan B, Prévot L, Roux B (2008) Can commercial digital cameras be used as multispectral sensors? A crop monitoring test. Sensors 8(11):7300-7322. DOI: https://doi.org/10.3390/s8117300
Logie GSJ, Coburn CA (2018) An investigation of the spectral and radiometric characteristics of low-cost digital cameras for use in UAV remote sensing. International Journal of Remote Sensing 39(15-16): 4891-4909. DOI: https://doi.org/10.1080/01431161.2018.1488297

Manzano JM, Narvaez JG, Castillo JG, Vásquez DR, Villada LG (2019) Analysis of Normalized Vegetation Index in Castile Coffee Crops, Using Mosaics of Multispectral Images Acquired by Unmanned Aerial Vehicle (UAV). International Conference on Applied Technologies. Springer, Cham. p546-559. DOI: https://doi.org/10.1007/978-3-030-42520-3_43

Mulla DJ (2013) Twenty five years of remote sensing in precision agriculture: Key advances and remaining knowledge gaps. Biosystems Engineering 114(4): 358-371. DOI: https://doi.org/10.1016/j.biosystemseng.2012.08.009

Murugan D, Garg A, Singh D (2017) Development of an Adaptive Approach for Precision Agriculture Monitoring with Drone and Satellite Data. IEEE Journal of Selected Topics in Applied Earth Observations and Remote Sensing 10(12): 5322-5328. DOI:

https://doi.org/10.1109/JSTARS.2017.2746185

Nijland W, Jong R, Jong SM, Wulder MA, Bater CW, Coopsa NC (2014) Monitoring plant condition and phenology using infrared sensitive consumer grade digital cameras. Agricultural and Forest Meteorology 184(2014): 98-106. DOI: https://doi.org/10.1016/j.agrformet.2013.09.007

Pozo SD, Rodríguez-Gonzálvez P, Hernández-López D, Felipe-García B (2014) Vicarious radiometric calibration of a multispectral camera on board an unmanned aerial system. Remote Sensing 6(3): 1918-1937. DOI: https://doi.org/10.3390/rs6031918

Putra BTW, Soni P (2017) Evaluating NIR-Red and NIRRed edge external filters with digital cameras for assessing vegetation indices under different illumination. Infrared Physics and Technology 81(2017): 148-156. DOI: https://doi.org/10.1016/j.infrared.2017.01.007

Wang C, Myint SW (2015) A Simplified Empirical Line Method of Radiometric Calibration for Small Unmanned Aircraft Systems-Based Remote Sensing. IEEE Journal of Selected Topics in Applied Earth Observations and Remote Sensing 8(5): 1876-1885. DOI: https://doi.org/10.1109/JSTARS.2015.2422716 